\title{
Cellules souches du système nerveux et réparation des lésions cérébrales
}

\section{$\mathbf{L}$} a découverte, il y a quelques années, de cellules souches ayant des capacités considérables de prolifération et de différenciation a fait progresser notre compréhension des mécanismes du développement du système nerveux. La possibilité de propager indéfiniment ces cellules en culture, ou de contrôler leur différenciation in vitro en neurones et cellules gliales, et les résultats spectaculaires d'expériences de transplantation dans le cerveau, ont de plus ouvert des perspectives encourageantes pour le traitement des lésions du système nerveux.

\section{Propriétés des cellules souches du système nerveux}

Une cellule souche est une cellule progénitrice indifférenciée qui d'une part s'autorenouvelle lorsqu'elle se divise, et d'autre part peut produire la plupart des types cellulaires du tissu auquel elle appartient. Des cellules souches existent dans de nombreux tissus, par exemple dans le système hématopoïétique, mais ce n'est qu'assez récemment que des cellules souches, capables de produire les principaux types cellulaires du système nerveux, neurones, astrocytes et oligodendrocytes, ont été identifiées, d'abord chez la souris puis chez l'homme [1, 2]. L'amélioration des techniques de culture a joué un rôle important dans cette découverte, avec notamment l'utilisation de facteurs mitogènes, le fibroblast growth factor (FGF) et l'epidermal growth factor (EGF) qui, lorsqu'ils sont ajoutés à fortes concentrations à des cultures dissociées de tissus nerveux, permettent la prolifération indéfinie des cellules souches [3, 4]. Le retrait de ces facteurs du milieu de culture entraîne leur différenciation progres- sive en neurones et cellules gliales [57] (figure 1).

Chez les rongeurs, la production de neurones se poursuit tout au long de la vie adulte dans deux régions du cerveau, le gyrus dentatus de l'hippocampe (GD) et la zone sous-ventriculaire du télencéphale (ZSV) (figure 2). Dans le reste du système nerveux, en revanche, la neurogenèse cesse pendant la vie embryonnaire ou peu de temps après la naissance. Plus tard, les neurones ne sont pas remplacés, même lorsqu'ils sont éliminés lors de maladies neurodégénératives ou d'accidents vasculaires. Des cellules ayant les propriétés de multipotentialité et d'autorenouvellement de cellules souches peuvent être cultivées à partir de cerveaux embryonnaires, ainsi qu'à partir du GD et de la ZSV de cerveaux adultes chez tous les mammifères [8-10]. De façon plus inattendue, des cellules souches ont

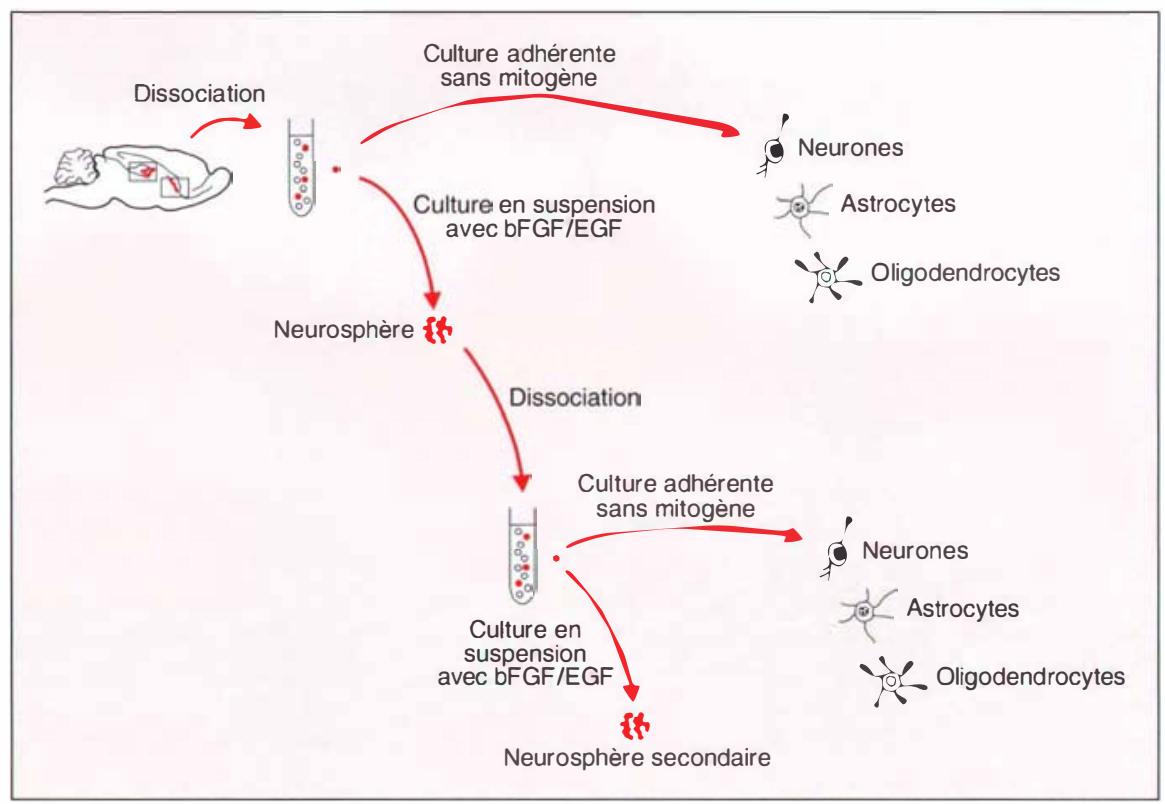

Figure 1. Identification de cellules souches du système nerveux en culture. En l'absence de marqueurs spécifiques, la présence de cellules souches dans un tissu nerveux ne peut être démontrée que par des expériences de culture $[5,6]$. Après dissociation du tissu, les cellules sont cultivées à faible densité en présence de facteurs mitogènes. Dans ces conditions, les cellules souches prolifèrent et chacune engendre un clone de cellules indifférenciées, ou neurosphère. Les neurosphères sont ensuite individuellement dissociées et cultivées dans différentes conditions. Lorsqu'elles sont cultivées en l'absence de facteur mitogène, elles se différencient en neurones, astrocytes et oligodendrocytes, démontrant la multipotentialité de la cellule souche à l'origine de la neurosphère. Lorsqu'elles sont cultivées à faible densité et en présence de facteurs mitogènes, elles produisent des neurosphères secondaires, démontrant la capacité d'autorenouvellement de la cellule souche d'origine. 


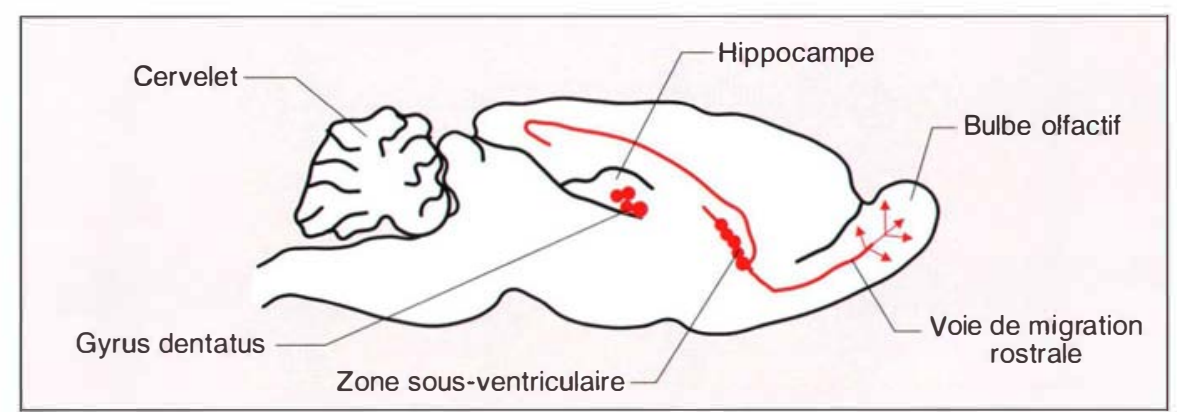

Figure 2. Régions neurogéniques du cerveau adulte. Alors que la neurogenèse cesse dans la plupart des régions du système nerveux pendant la vie embryonnaire ou peu après la naissance, deux régions du cerveau de la souris, le gyrus dentatus (GD) et la zone sous-ventriculaire (ZSV), contiennent des cellules souches qui restent actives pendant la vie adulte. Les cellules souches de la zone sous-ventriculaire produisent des précurseurs neuronaux qui migrent sur une longue distance le long de la voie de migration rostrale avant de se différencier en neurones dans le bulbe olfactif. La présence de cellules souches actives dans l'hippocampe a été également démontrée chez I'homme adulte [10].

également été isolées à partir de nombreuses régions du système nerveux adulte ne présentant aucune activité neurogénique in vivo, comme la moelle épinière, ce qui suggère qu'elles persistent normalement chez l'adulte dans un état quiescent, ou avec une activité seulement gliogénique [11, 12]. Faute de marqueurs moléculaires spécifiques, l'identification in situ des cellules souches du système nerveux s'est avérée difficile ([13, 14] et $\left.\mathrm{m} / \mathrm{s} 1998, n^{\circ} 12, p .1453\right)$, et leur purification, qui faciliterait grandement leur étude, n'est actuellement pas réalisable.

L'absence de neurogenèse et de capacité de régénération dans la plupart des tissus du svstème nerveux adulte, même s'ils contiennent des cellules souches, peut s'expliquer soit par l'absence des signaux inducteurs de différenciation neuronale qui sont présents che\% l'embryon, soit, si ces signaux persistent che» l'adulte, par la présence de facteurs inhibiteurs de la neurogenèse. Les signaux réglant in situ l'activité des cellules souches, et notamment la neurogenèse, ne sont pas encore connus.

\section{Plasticité de la différenciation des cellules souches du système nerveux}

Au cours du développement embiyonnaire, des neurones de pliénotypes dans les différentes régions du système nerveux. Pour tester le potentiel de différenciation des cellules souches de façon plus complète que cela n'est possible en culture, des cellules propagées in vitro en présence de facteurs mitogènes ont été transplantées dans des régions neurogéniques du cerveau. Les résultats de ces expériences, qui ont surpris par la grande diversité des types cellulaires produits in vivo par les cellules souches, ont permis de conclure que le devenir des cellules greffées est déterminé par les signaux locaux auxquels elles sont exposées, plutôt que par des propriétés intrinsèques. Les cellules transplantées se mélangent aux progéniteurs endogènes, migrent et participent au développement normal du système nerveux de l'animal hôte en se différenciant en types neuronaux et gliaux normalement présents dans la région de la greffe. Ainsi des cellules progénitrices provenant de l'hippocampe de souris adultes et transplantées dans la ZSV de souris adultes, dans la rétine ou le cervelet de souriceaux nouveau-nés peuvent se différencier respectivement en tvpes neuronaux spécifiques du bulbe olfactif, de la rétine, et du cervelet $[15,16]$ (Jiguie 3). En faveur d'une grande plasticité de la différenciation des cellules souches, des expériences ont montré qu'elles peuvent même produire des cellules non nerveuses, en l'occurrence hématopoïétiques, lorsqu'elles sont soumises aux conditions de différenciation adéquates [17]. Il faut cependant noter que d'autres travaux concluent plutôt à l'existence de certaines restrictions dans la capacité des cellules souches à adopter certains phénotypes [16].

Les propriétés remarquables des cellules souches du système nerveux, étudiées initialement chez les rongeurs, ont rapidement suggéré que ces cellules pouvaient être d'excellents instruments pour le traitement de maladies du système nerveux. Premièrement, leur capacité de se différencier en neurones, astrocytes et oligodendrocytes après transplantation dans le cerveau, permet d'envisager leur utilisation dans des dégénérescences neuronales mais aussi des maladies démyélinisantes. Deuxièmement, leur capacité de se différencier de façon appropriée, y compris dans des régions distinctes de leur région d'origine, permet d'utiliser les mêmes cellules pour des maladies affectant différentes populations neuronales. Troisièmement, les cellules souches, qui peuvent être propagées en culture sans altération de leurs propriétés, sont en principe une source abondante de cellules utilisables pour la transplantation. Finalement, les cellules souches peuvent êtes manipulées génétiquement in vitro et servir de véhicule pour l'expression de gènes étrangers dans le cerveau, par exemple des gènes favorisant la survie, la migration, la différenciation ou la croissance ax()nale des cellules transplantées.

\section{Perspectives thérapeutiques de l'utilisation des cellules souches du système nerveux}

Depuis quelques années, de nombreux travaux sont consacrés à l'étude de cellules progénitrices humaines isolées à partir de cerveaux fotaux. Comme les cellules souches de souris, ces cellules proliférent indéfiniment en culture en présence de FGF et de EGF, et donnent naissance à des neurones, des astrocytes et des oligodendrocytes lorsqu'elles sont cultivées dans des conditions favorisant leur différenciation $[18,19]$. Des expériences récentes de transplantation dans le 


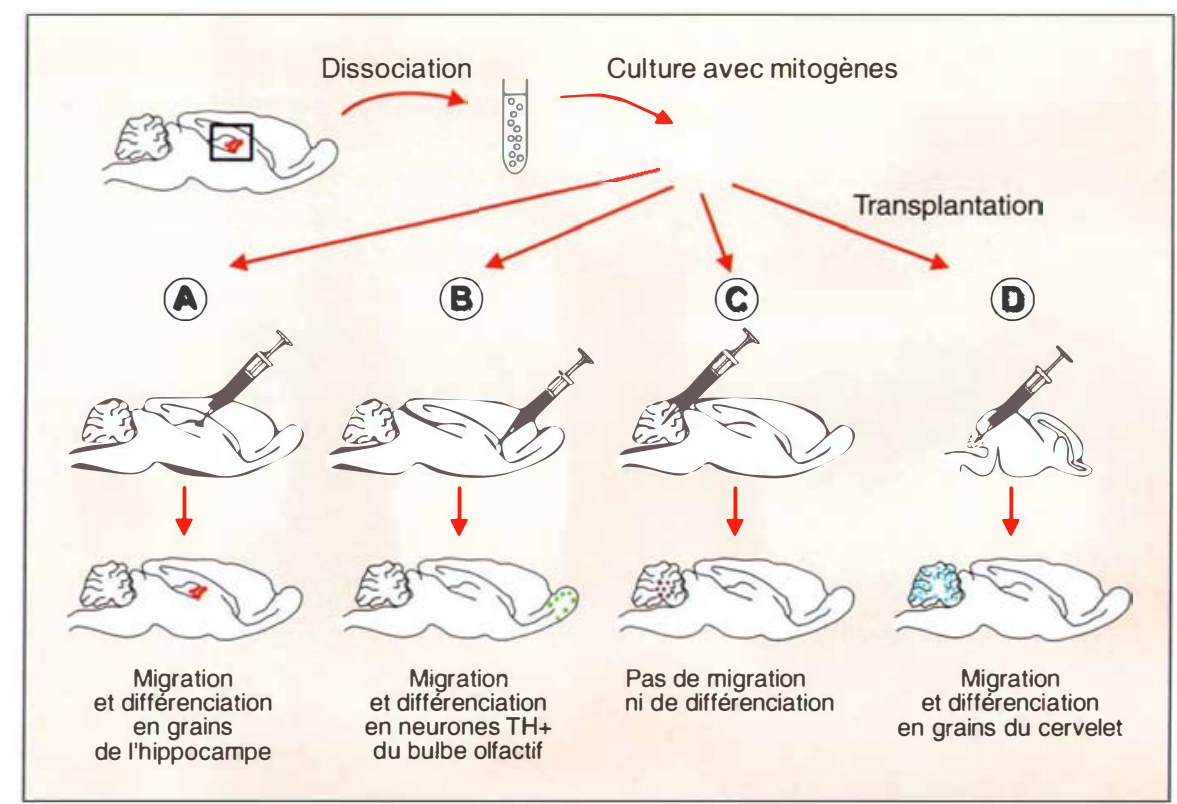

Figure 3. Plasticité des cellules souches du système nerveux. La plasticité de différenciation des cellules souches a été démontrée par des expériences de transplantation. Des cellules progénitrices ont été isolées de l'hippocampe de rats adultes, puis cultivées en présence de mitogènes et enfin transplantées dans différentes régions du cerveau de rats nouveau-nés ou adultes [15]. Lorsqu'elles sont greffées dans des régions neurogéniques, telles que le gyrus dentatus (GD) (A) et la zone sous-ventriculaire (ZSV) (B) d'animaux adultes, et le cervelet de nouveau-nés (D), les cellules transplantées migrent et se différencient de façon caractéristique de la région dans laquelle elles ont été greffées, par exemple dans le cas de précurseurs se différenciant dans le bulbe olfactif (B), en neurones exprimant la tyrosine hydroxylase (TH), un type neuronal absent de l'hippocampe. La migration et le mode de différenciation des cellules souches sont donc largement dictés par des signaux de l'environnement dans lequel ces cellules se différencient. Les cellules greffées dans des régions non neurogéniques, comme le cervelet d'animaux adultes (C) ne migrent pas et ne se différencient pas en neurones, suggérant que ces signaux ne sont présents que transitoirement lors de la neurogenèse.

cerveau de rongeurs ont montré que ces cellules progénitrices humaines ont les mêmes propriétés de plasticité que les cellules souches de souris. Elles peuvent répondre aux signaux locaux, qui assurent non seulement leur survie, leur intégration et leur migration dans le tissu hote, mais aussi leur différenciation en types cellulaires correspondant au stade de développement et à la région de la transplantation [20-22]. Ainsi, des cellules souches humaines provenant de télencéphale foetal et greffées clans le cervelet de souriceaux nouveau-nés portant la mutation meander tail peuvent remplacer efficacement les neurones cérébel- par exemple le cas dans le cortex cérébral où, à la suite de la mort ciblée des neurones pyramidaux de certaines couches corticales, des cellules progénitrices transplantées se différencient spécifiquement en neurones du type manquant, et uniquement dans les régions du cortex cérébral et les couches affectées [25].

Le succès des greffes de cellules nerveuses dépend également du traitement subi par les cellules transplantées, et notamment de leurs conditions de culture. Dans la perspective du traitement de maladies neurologiques par transplantation cellulaire, le fait de pouvoir cultiver les cellules avant de les transplanter est extrêmement important. En effet, une des principales difficultés rencontrées dans le traitement de patients atteints de maladie de Parkinson par transplantation de cellules foetales est le nombre restreint de neurones dopaminergiques - le type cellulaire affecté chez ces patients qui peuvent être obtenus à partir de cerveaux foetaux. La possibilité de propager en culture les cellules progénitrices avant de les transplanter chez des patients constituerait donc un progrès considérable. Cette approche pose cependant un problème, car les cultures réalisées en présence de EGF et de FGF contiennent une large fraction de cellules très immatures qui ne produisent pas beaucoup de neurones lorsqu'elles sont transplantées dans une région non neurogénique du cerveau adulte [26] (figure 3). En revanche, les résultats des greffes sont meilleurs quand des cellules plus mûres sont utilisées. Ainsi, la différenciation de cellules souches en culture après leur amplification permet après transplantation à un grand nombre de neurones de survivre à long terme et de s'intégrer fonctionnellement [27] (figure 4). L'utilisation d'une telle stratégie de différenciation in vitre de cellules souches dans le traitement de maladies neurologiques implique que les signaux contrôlant la différenciation des types cellulaires affectés clans la maladie soient identifiés. Il s'agit d'un défi considérable pour les chercheurs travaillant clans ce clomaine, mais des progrès encourageants ont été récemment obte- 


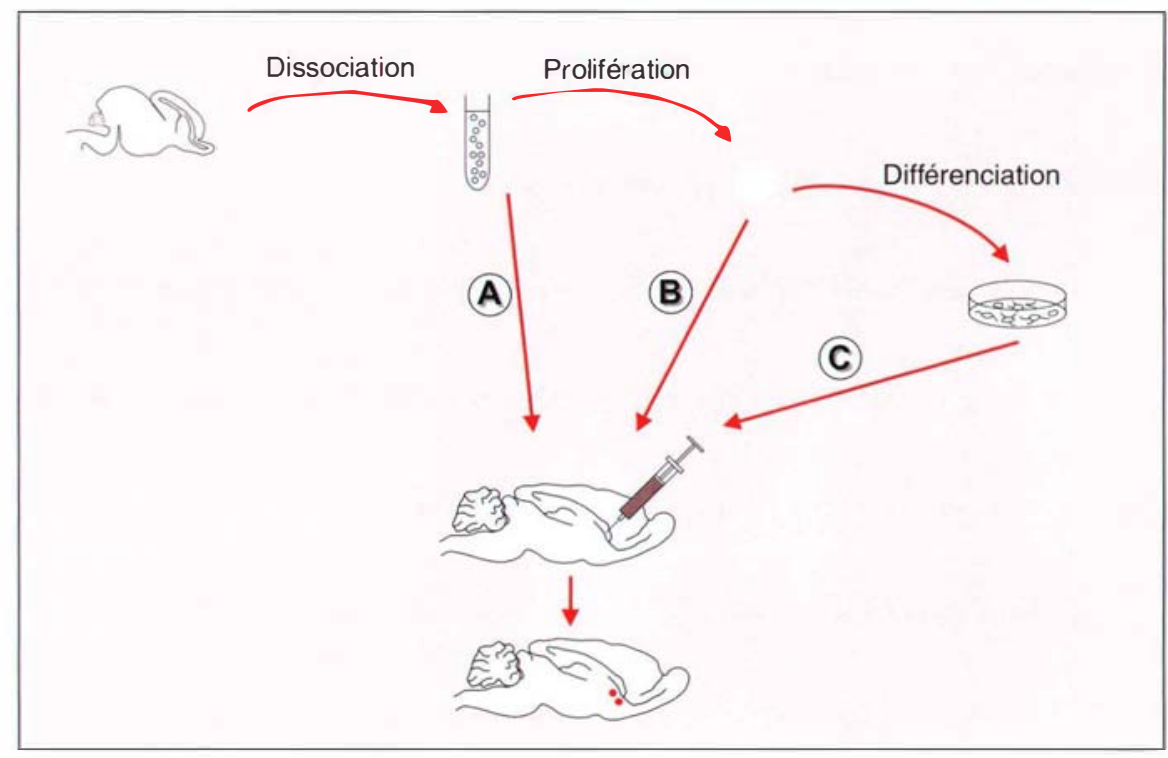

Figure 4. Réparation de lésions du système nerveux par transplantation de cellules progénitrices. Pour tester la capacité de cellules progénitrices de réparer des lésions du système nerveux, des cellules d'origine murine ou humaine ont été transplantées dans le cerveau d'animaux chez lesquels des neurones dopaminergiques du mésencéphale ou des neurones pyramidaux du cortex cérébral ont été sélectivement détruits, afin de créer des modèles de maladies neurodégénératives. Lorsque les cellules greffées ont été directement dissociées de cerveaux embryonnaires (A), elles s'intègrent correctement dans le tissu hôte et se différencient en neurones de même type que ceux ayant dégénéré [32]. L'utilisation de tissus fœtaux présente cependant l'inconvénient de ne disposer que de petites quantités de cellules à greffer, qui sont de plus de composition hétérogène et peu reproductible. L'amplification de cellules progénitrices en culture en présence de facteurs mitogènes (B) permet d'obtenir des cellules à greffer en beaucoup plus grandes quantités et de composition mieux définie. Cependant, les greffes de cellules très immatures ne résultent généralement qu'en un petit nombre de cellules greffées survivant à long terme et se différenciant dans le cerveau hôte [26]. En revanche, si des cellules progénitrices qui ont été propagées en présence de mitogènes sont d'abord différenciées in vitro dans le type cellulaire approprié (C), par exemple en neurones dopaminergiques mésencéphaliques dans le cas de transplantations dans le cerveau d'animaux modèles de maladie de Parkinson, le résultat de la greffe peut être largement amélioré [27].

nus dans le contrôle de la différenciation in vitro de neurones dopaminergiques mésencéphaliques [27, 28].

\section{Stratégies alternatives \\ pour le traitement \\ de maladies neurologiques}

Malgré le grand espoir suscité par les cellules souches pour le traitement de maladies neurologiques, leur utilisation n'est pas sans inconvénients, liés en particulier au fait que ces cellules doivent être isolées à partir de
ES, puis transplantés dans le cerveau, s'implantent efficacement et se différencient comme les cellules hôtes voisines [30], et ont pu par exemple corriger un défaut de myélinisation chez des souris mutantes [31].

Pour éviter plus généralement les problèmes liés aux transplantations, les stratégies de remplacement cellulaire pourraient dans le futur reposer sur l'activation des programmes neurogéniques ou gliogéniques des cellules souches endogènes, grâce à l'action des facteurs extrinsèques introduits par exemple par transfert de gène ou par injection. Pour que certaines des stratégies envisagées utilisant les cellules souches dans le traitement de maladies neurologiques se concrétisent, des progrès importants devront être réalisés dans les connaissances fondamentales sur la biologie de ces cellules, et notamment sur les mécanismes de leur différenciation dans les multipes types cellulaires qui composent le système nerveux

\section{Carlos Parras \\ Marta Nieto \\ François Guillemot}

Institut de génétique et de biologie moléculaire et cellulaire, Cnrs/Inserm/Université Louis-Pasteur, BP 163, 67404 Illkirch Cedex, CU de Strasbourg, France.

\section{RÉFÉRENCES}

1. McKay R. Stem cells in the central nervous system. Science 1997; 276: 66-71.

2. Gage FH. Mammalian neural stem cells Science 2000; 287: 1433-9.

3. Richards LJ, Kilpatrick TJ, Bartlett PF. De novo generation of neuronal cells from the adult brain. Proc Natl Acad Sci USA 1992; $89: 8591-5$.

4. Reynolds BA, Weiss S. Generation of neurons and astrocytes from isolated cells of the adult mammalian central nervous system. Science 1992; 255: 1707-10.

5. Gritti A, Parati EA, Cova L, et al. Multipotential stem cells from the adult mouse brain proliferate and self-renew in response to basic fibroblast growth factor. I Neurosci 1996; 16: 1091-100.

6. Reynolds BA, Weiss S. Clonal and population analyses demonstrate that an EGF-responsive mammalian embryonic CNS precursor is a stem cell. Dev Biol 1996; $175: 1-13$.

$\mathrm{m} / \mathrm{s} n^{\circ} 6-7$, vol. 16, juin-juillet 2000 


\section{RÉFÉRENCES}

7. Guillemot F. Signaux extracellulaires et programmes transcriptionnels contrôlant la neurogenèse. Med Sci 2000; 16: 159-63.

8. Lois C, Alvarez-Buylla A. Proliferating subventricular zone cells in the adult mammalian forebrain can differentiate into neurons and glia. Proc Natl Acad Sci USA 1993; $90: 2074-7$.

9. Morshead CM, Reynolds BA, Craig CG, et al. Neural stem cells in the adult mammalian forebrain: a relatively quiescent subpopulation of subependymal cells. Neuron $1994 ; 13$ : 1071-82.

10. Eriksson PS, Perfilieva E, Bjork-Eriksson T, et al. Neurogenesis in the adult human hippocampus. Nat Med 1998; 4: 1313-7.

11. Kehl LJ, Fairbanks CA, Laughlin TM Wilcox GL, et al. Neurogenesis in postnata rat spinal cord: a study in primary culture. Science 1997 ; 276 : 586-9.

12. Palmer TD, Markakis EA, Willhoite AR Safar F, Gage FH. Fibroblast growth factor-2 activates a latent neurogenic program in neural stem cells from diverse regions of the adult CNS. $J$ Neurosci 1999; 19:8487-97.

13. Johansson CB, Momma S, Clarke DL Risling M, Lendahl U, Frisen, J. Identification of a neural stem cell in the adult mammalian central nervous system. Cell 1999 $96: 25-34$.

14. Doetsch F, Caille I, Lim DA, Garcia-Verdugo JM, Alvarez-Buylla A. Subventricular zone astrocytes are neural stem cells in the adult mammalian brain. Cell 1999; 97: 703 16.

15. Suhonen JO, Peterson DA, Ray J, Gage FH. Differentiation of adult hippocampusderived progenitors into olfactory neurons in vivo. Nature $1996 ; 383: 624-7$.

16. Takahashi M, Palmer TD, Takahashi J Gage FH. Widespread integration and survi- val of adult-derived neural progenitor cells in the developing optic retina. Mol Cell Neu rosci 1998 ; 12 : 340-8.

17. Bjornson CR, Rietze RL, Reynolds BA Magli MC, Vescovi AL. Turning brain into blood: a hematopoietic fate adopted by adult neural stem cells in vivo. Science 1999 . 283: $534-7$

18. Carpenter MK, Cui X, Hu ZY, et al. In vitro expansion of a multipotent population of human neural progenitor cells. Exp Neurol 1999; 158: 265-78.

19. Vescovi AL, Parati EA, Gritti A, et al. Isolation and cloning of multipotential stem cells from the embryonic human CNS and establishment of transplantable human neural stem cell lines by epigenetic stimulation. Exp Neurol 1999; 156: 71-83.

20. Brüstle O, Choudhary K, Karram K, et al Chimeric brains generated by intraventricular transplantation of fetal human brain cells into embryonic rats. Nat Biotechnol $1998 ; 16: 1040-4$

21. Flax JD, Aurora S, Yang C, et al. Engraf table human neural stem cells respond to developmental cues, replace neurons, and express foreign genes. Nat Biotechnol 1998 ; 16: 1033-9.

22. Fricker RA, Carpenter MK, Winkler C, Greco C, Gates MA, Bjorklund A. Site-speciGreco C, Gates MA, Bjorklund A. Site-speci-
fic migration and neuronal differentiation of human neural progenitor cells after transplantation in the adult rat brain. $J \mathrm{Neu}$ rosci $1999 ; 19$ : 5990-6005.

23. Gage FH, Coates PW, Palmer TD. Survival and differentiation of adult neuronal progenitor cells transplanted to the adult brain. Proc 24. Suhonen JO, Peterson DA, Ray J, Gage
FH. Differentiation of adult hippocampusderived progenitors into olfactory neurons in vivo. Nature 1996; 383: 624-7.
25. Snyder EY, Yoon C, Flax JD, Macklis JD. Multipotent neural precursors can differentiate toward replacement of neurons undergoing targeted apoptotic degeneration in adult mouse neocortex. Proc Natl Acad Sci USA 1997; 94: 11663-8.

26. Svendsen CN, Clarke DJ, Rosser AE Dunnett SB. Survival and differentiation of rat and human epidermal growth factorresponsive precursor cells following grafting into the lesioned adult central nervous system. Exp Neurol 1996; 137: 376-88.

27. Studer L, Tabar V, McKay RD. Transplantation of expanded mesencephalic precursors leads to recovery in parkinsonian rats. Nat Neurosci $1998 ; 1$ : $290-5$.

28. Wagner J, Akerud P, Castro DS, et al. Induction of a midbrain dopaminergic phenotype in Nurrl-overexpressing neural stem cells by type 1 astrocytes. Nat Biotechnol $1999 ; 17: 653-9$.

29. Svendsen NC and Smith AG. New prospects for human stem-cell therapy in the nervous system. Trends Neurosci 1999; 22 : 357-64.

30. Brüstle O, Spiro AC, Karram K, Choudhary $K$, Okabe S, McKay RD. In vitro-generated neural precursors participate in mammalian brain development. Proc Natl Acad Sci USA 1997; 94 : 14809-14.

31. Brüstle $\mathrm{O}$, Jones $\mathrm{KN}$, Learish $\mathrm{RD}$, et al. Embryonic stem cell-derived glial precursors: a source of myelinating transplants. Science $1999 ; 285$ : $754-6$

32. Sheen VL, Macklis JD. Targeted neocortical cell death in adult mice guides migration and differentiation of transplanted embryonic neurons. $J$ Neurosci $1995 ; 15: 8378-92$.

\section{TIRÉS À PART}

F. Givillemot.

\section{IFR Broca-Sainte-Anne}

L'IFR Broca-Sainte-Anne sur les maladies du Système Nerveux Central regroupe plusieurs Unités de Recherches Inserm et les chercheurs cliniciens de plusieurs services du Centre Hospitalier Spécialisé Sainte-Anne et de l'Hôpital Broca. Les principaux axes de recherches actuels sont : (1) la génétique moléculaire des affections psychiatriques ( $y$ compris toxicomanies) et des accidents vasculaires cérébraux, (2) les mécanismes cellulaires de l'épilepsie, (3) la neuro-oncologie, (4) la physiopathologie du vieillissement cérébral, (5) le développement de nouvelles classes de médicaments neurologiques et psychiatriques. En outre, le site souhaite développer des recherches dans le domaine de I'imagerie fonctionnelle appliquée aux affections psychiatriques et neurologiques.

Afin de renforcer ces axes et la synergie entre recherche fondamentale et activités cliniques, I'IFR se propose d'accueillir dans des locaux vacants du site des chercheurs isolés ou en équipe s'intéressant à ces thématiques.

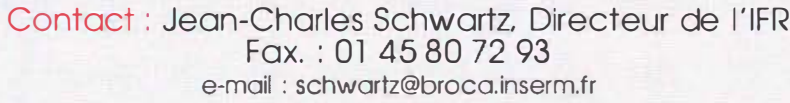

